2. Korth J, Wilde B, Dolff S, et al. SARS-CoV-2-specific antibody detection in healthcare workers in Germany with direct contact to COVID-19 patients. J Clin Virol 2020;128:104437.

3. Bampoe S, Lucas DN, Neall G, et al. A cross-sectional study of immune seroconversion to SARS-CoV-2 in frontline maternity health professionals. Anaesthesia 2020. doi: 10.1111/anae.15229.

4. Garcia-Basteiro AL, Moncunill G, Tortajada M, et al. Seroprevalence of antibodies against SARS-CoV-2 among healthcare workers in a large Spanish reference hospital. Nat Comm 2020;11. doi: 10.1038/s41467-020-17318-x.

5. Patel MM, Thornsburg NJ, Stubblefield WB, et al. Change in antibodies to SARS-CoV-2 over 60 days among healthcare personnel in Nashville, Tennessee. JAMA Network 2020. doi: 10.1001/jama.2020.18796.
6. Blairon L, Mokrane S, Wilmet A, Desilly G, et al. Large-scale, molecular, and serological SARS-CoV-2 screening of healthcare workers in a 4-site public hospital in Belgium after COVID-19 outbreak. J Infect 2020. doi: 10.1016/ j.jinf.2020.07.033.

7. Martin C, Montesinos I, Dauby N, et al. Dynamics of SARS-CoV-2 RT-PCR positivity and seroprevalence among high-risk healthcare workers and hospital staff. J Hosp Infect 2020. doi: 10.1016/j.jhin.2020.06.028.

8. Iversen K, Bundgaard H, Hasselbalch RM, et al. Risk of COVID-19 in healthcare workers in Denmark: an observational cohort study. Lancet 2020. doi: 10.1016/S1473-3099(20)30589-2.

9. Lackermair K, William F, Grzanna N, et al. Infection with SARS-CoV-2 in primary care healthcare workers assessed by antibody testing. Fam Pract 2020. doi: 10.1093/fampra/cmaa078.

\title{
Silver linings of the coronavirus disease 2019 (COVID-19) pandemic from an infection prevention and control perspective
}

\author{
Kara K. Tsang BHSc${ }^{1}$ (1), Dominik Mertz MD², Cindy O’Neill MLT³ and Sarah Khan MD \\ ${ }^{1}$ Department of Biochemistry and Biomedical Sciences, McMaster University, Hamilton, Ontario, Canada, ${ }^{2}$ Department of Medicine, Infection Prevention and \\ Control, McMaster University, Hamilton Health Sciences, Hamilton, Ontario, Canada, ${ }^{3}$ Hamilton Health Sciences, Hamilton, Ontario, Canada and ${ }^{4}$ Department of \\ Pediatrics, Infection Prevention and Control, McMaster University, Hamilton Health Sciences, Hamilton, Ontario, Canada
}

To the Editor-As a response to the evolving information about the coronavirus disease 2019 (COVID-19) pandemic, health agencies and organizations have been updating guidelines for infection prevention and control. McMullen et $\mathrm{al}^{1}$ made some predictions about increasing and decreasing hospital-acquired infection rates due to the crisis care for COVID-19. Despite the numerous infection control challenges posed by severe acute respiratory coronavirus virus 2 (SARS-CoV-2) in acute-care facilities, in some ways, we have been able to identify some "silver linings" or opportunities for improved infection control practices amidst the pandemic.

\section{Quality assurance of personal protective equipment (PPE) practices}

Previous to the COVID-19 pandemic, our hospital followed the Public Health Ontario Provincial Infectious Diseases Advisory Committee's recommendations for different masks and uses, which did not delineate which ASTM mask levels were appropriate. The American Society for Testing and Materials International is a standards organization that develops and publishes consensus technical standards used in the production and testing of personal protective equipment. ${ }^{2}$ Due to the global shortage of PPE supplies, we were able to vet all of our PPE stocks to assess their quality. As part of this audit, we uncovered facemasks in widespread circulation that were not ASTM rated for fluid resistance; these were subsequently removed from our clinical areas. Furthermore, as part of this detailed review of our PPE supplies, we developed standardization in ASTM mask levels appropriate for clinical areas based on the likelihood of facial fluid splash, and for most areas, an ASTM level 1 mask was

Author for correspondence: Sarah Khan, E-mail: khan259@mcmaster.ca

Cite this article: Tsang KK, et al. (2021). Silver linings of the coronavirus disease 2019 (COVID-19) pandemic from an infection prevention and control perspective. Infection Control \& Hospital Epidemiology, 42: 1408-1409, https://doi.org/10.1017/ice.2020.1310 considered acceptable. This exercise would never have been conducted if the pandemic had not resulted in the development of a PPE task force to review our current inventory and our standard stocking practices.

Concerning the empowerment of staff in their PPE and choices, as part of the pandemic PPE training, all staff were instructed on the process of a point-of-care risk assessment. Despite this being a longstanding aspect of orientation for all staff, only in the pandemic has this become regular verbiage among the staff, indicating effective uptake of basic infection control principles that had previously remained aloof concepts. Similarly, a longstanding principle is that any aerosol-generating medical procedure (AGMP) requires a mask plus eye protection routinely, and a N95 respirator is also required if COVID-19 is suspected. ${ }^{3}$ Furthermore, eye protection has not been generally used within operating rooms, intensive care units (ICU), and emergency departments (EDs) across the province for multiple AGMPs, including intubations (personal communication with Chris Simpson, Queen's University). With the pandemic, however, uptake of eye protection for such scenarios has significantly improved. Similarly, environmental controls that should have been in place, including plexiglass or a physical barrier ${ }^{4}$ to protect staff from droplet transmission from any virus exposure when encountering unscreened patient populations were implemented. Similarly, screening for febrile respiratory tract infection is performed more systematically and is now the determinant of implementing additional precautions. In contrast, screening may not have previously been as methodical, and precautions often were initiated only if a pathogen was identified from a nasopharyngeal swab.

\section{Increased hand hygiene compliance rates}

In addition to the new systematic guidelines for PPE, we observed improved hand hygiene compliance rates during the COVID-19

(c) The Author(s), 2020. Published by Cambridge University Press on behalf of The Society for Healthcare Epidemiology of America. This is an Open Access article, distributed under the terms of the Creative Commons Attribution licence (http://creativecommons.org/licenses/by/4.0/), which permits unrestricted re-use, distribution, and reproduction in any medium, provided the original work is properly cited. 
Table 1. Hand Hygiene Compliance for Moments 1 Through 4 Between January and April 2020

\begin{tabular}{|c|c|c|c|c|}
\hline Type of Indication & $\begin{array}{c}\text { September } 2018-2019 \\
\text { Compliance, } \\
\text { No. (\%) }\end{array}$ & $\begin{array}{c}\text { January-April } 2020 \\
\text { Compliance, } \\
\text { No. (\%) }\end{array}$ & $\chi^{2}$ & $\begin{array}{c}P \\
\text { Value }\end{array}$ \\
\hline Moment 1. Before initial patient-to-patient environmental contact & $3,606 / 4,398(82.0)$ & $677 / 793(85.4)$ & 5.32 & .02 \\
\hline Moment 2. Before aseptic procedure & $284 / 557(51)$ & $30 / 107(28.0)$ & 35.64 & $<.00001$ \\
\hline Moment 3. After body fluid exposure risk & $542 / 816(66.4)$ & $88 / 145(60.7)$ & 1.79 & .18 \\
\hline Moment 4. After patient-to-patient environmental contact & $7,432 / 8,673(85.4)$ & $1,400 / 1,588(88.2)$ & 6.83 & .009 \\
\hline
\end{tabular}

pandemic. Between January and April 2020, hand hygiene compliance for Moments 1 and 4 have improved compared to September 2018-December 2019 (Table 1). We suspect that the COVID-19 pandemic has introduced additional concern among healthcare workers, who have become more likely to comply with proper hand hygiene practices for their own safety and that of their patients.

\section{Decrease in rates of hospital-acquired infections}

In terms of hospital-acquired infections, methicillin-resistant Staphylococcus aureus (MRSA), vancomycin-resistant Enterococci (VRE), extended-spectrum B-lactamase (ESBL), and carbapenemaseproducing Enterobacteriaceae (CRE) infection rates per 1,000 patient days in 2020 (January-April) were lower compared to the previous year. Specifically, at one of our hospital sites, the rates per 1,000 patient days for VRE and ESBL bacteremia infections have also decreased compared to the previous year. Thus, the decrease in infection rates could also be attributed to lower bed occupancy between January and April 2020. Lastly, as part of continuous quality improvement huddles in wards, the environmental aids and cleaning staff are becoming increasingly part of infection prevention conversations. Data on cleaning auditing results are being presented more often, and staff have a heightened awareness of the potential for fomite spread, despite it being a small component of COVID transmissions. Ontario health guidelines around optimization of physical distancing, as it pertains to SARSCoV-2 control, have recommended against the use of 4-bed ward rooms, which has been a source for patient-to-patient transmissions and outbreaks of other healthcare-associated infections due to crowding and to limited ability to clean between patient equipment and belongings. This change may contribute to reductions in the spread of antimicrobial-resistant organisms and to other healthcare-associated infection outcomes.

In summary, despite the global challenges posed by the COVID-19 pandemic for infection preventionists, some valuable short-term gains have been made during this time. However, as hospitals now being to 'reopen' to more typical volumes, we may lose ground in areas where we have been able to improve practices. For now, we should celebrate these small wins, and we should aim to continue this culture shift in the minds of frontline workers, prioritizing infection control, PPE practices, and environmental resources that have been put in place. It will be important to be mindful of the potential for 'mask fatigue' and for efforts to relax as these public health efforts 'flatten the curve.'

Acknowledgments. The authors would like to acknowledge the work of the infection prevention and control team at HHS who contributed the data, and have worked tirelessly throughout this pandemic to keep our patients safe.

Financial support. No financial support was provided relevant to this article.

Conflicts of interest. All authors report no conflicts of interest relevant to this article.

\section{References}

1. McMullen KM, Smith BA, Rebmann T. Impact of SARS-CoV-2 on hospital acquired infection rates in the United States: predictions and early results. Am J Infect Control 2020;48:1409-1411.

2. ASTM Standards and COVID-19. ASTM website. https://www.astm.org/ COVID-19/. Accessed July 16, 2020.

3. Volling C, Salvadori M, Brooks J. COVID-19: masks and face shields for duration of acute healthcare setting shifts. Government of Canada website. https://www.canada.ca/en/public-health/services/diseases/2019novel-coronavirus-infection/health-professionals/technical-brief-masking-faceshields-full-duration-shifts-acute-healthcare-settings.html. Published April 15, 2020. Accessed October 29, 2020.

4. Rational use of personal protective equipment for coronavirus disease (COVID-19): interim guidance-2-Recommendations for optimizing the availability of PPE. World Health Organization website. https://apps. who.int/iris/handle/10665/331498. Published 2020. Accessed October 29, 2020 . 